\title{
CONCEITO AMPLIADO DE SAÚDE NA FORMAÇÃO DOS PROFISSIONAIS DE EDUCAÇÃO FÍSICA
}

\section{Luciane Peter Grillo}

Doutora em Ciências Aplicadas à Pediatria pela Universidade Federal de São Paulo - Unifesp. Pósdoutora em Epidemiologia, pela Universidade Federal de Pelotas - UFPEL. Docente do Programa de Mestrado em Saúde e Gestão do Trabalho da Universidade do Vale do Itajaí/SC, Brasil.

\section{Leonardo Sartório Ibarra}

Mestre em Saúde e Gestão do Trabalho, pela Universidade do Vale do Itajaí - Univali, Itajaí/SC, Brasil.

\section{Tatiana Mezadri}

Doutora em Ciências dos Alimentos, Sevilha, Espanha. Docente do Programa de Mestrado em Saúde e Gestão do Trabalho, pela Universidade do Vale do Itajaí - Univali, Itajaí/SC, Brasil.
RESUMO: O estudo teve como objetivo avaliar 81 profissionais de educação física egressos de uma universidade comunitária sobre o conhecimento do conceito ampliado de saúde na sua formação. Trata-se de uma pesquisa quantitativa por meio da aplicação de um questionário online. Os resultados mostraram a predominância de profissionais do sexo masculino (56\%), faixa etária de 20 a 25 anos (55\%), carga horária de trabalho semanal de mais de 20 horas (67\%), renda de até $\mathrm{R} \$ 2.000,00$ mensais (78\%), dos quais $14 \%$ não apresentavam conhecimento do conceito ampliado de saúde. Além disso, verificou-se uma associação significativa entre a modalidade de formação e o conceito ampliado de saúde. Em vista dos achados observados, torna-se necessário consolidar a formação deste profissional na saúde coletiva para inserção nas equipes multiprofissionais.

PALAVRAS-CHAVE: Educação física e treinamento; Educação profissional em saúde pública; Saúde pública.

\section{EXTENDED HEALTH CONCEPT IN THE FORMATION OF PHYSICAL EDUCATION PROFESSIONALS}

ABSTRACT: Current analysis aimed at evaluating 81 professionals in physical education recently graduated with regard to their knowledge on extended health concept in their formation, by means of a quantitative research with an online questionnaire. Results revealed the predominance of male professionals (56\%), age bracket $20-25$ years (55\%), weekly work load of more than $20 \mathrm{~h}(67 \%)$, and an monthly income of $R \$ 2,000$ (78\%) Fourteen percent did not know anything on extended health concept. There was a significant association between training modality and extended health concept. Results showed the need for the consolidation in the formation of professionals in community health for insertion in multiprofessional teams.

KEY WORDS: Physical education and training; Professional education in public health; Public health.

\section{INTRODUÇão}

Com a implantação das diretrizes curriculares para os cursos de graduação, os currículos da área da saúde deveriam procurar uma articulação com o Sistema Único de Saúde (SUS), utilizando uma concepção ampliada de saúde. Entende-se, no presente estudo, como conceito ampliado de saúde: "A saúde resultante das condições de alimentação, habitação, educação, renda, meio ambiente, trabalho, transporte, emprego, lazer, liberdade, acesso e posse da terra e acesso aos
Autor correspondente

Luciane Peter Grillo

grillo@univali.br 
serviços de saúde. Sendo assim, é principalmente resultado das formas de organização social, de produção, as quais podem gerar grandes desigualdades nos níveis de vida"1. Desta forma, a integralidade passa a ter importância fundamental, uma vez que favorece a ampliação e o desenvolvimento da dimensão cuidadora e propicia maior comprometimento dos profissionais com o seu trabalho, favorecendo práticas inovadoras, fortalecendo os processos de educação permanente ${ }^{2}$.

A formação do profissional de educação física (EF), conforme resolução $\mathrm{n}^{0}$ 07/2004, de 31 de março de 2004, que institui as Diretrizes Curriculares Nacionais (DCNs) para os cursos de graduação em EF, em nível superior de graduação plena, deve ser concebida, planejada, operacionalizada e avaliada, visando à aquisição e o desenvolvimento de competências e habilidades específicas que contemplem a perspectiva da promoção da saúde nas diferentes esferas de atuação destes profissionais. Propõem, ainda, a formação de um perfil profissional voltado ao entendimento do contexto social dos indivíduos e comunidades para nele intervir profissionalmente com a sua especialidade acadêmica e com a ampliação do conhecimento, adotar hábitos saudáveis ${ }^{3}$.

Na saúde, o profissional de EF está inserido no Serviço de Atenção Básica ao compor as equipes do Núcleo de Apoio à Saúde Família (NASF), de acordo com as ações específicas priorizadas pela Política Nacional de Promoção da Saúdé, criada em 2006, que inclui a prática corporal e a atividade física nas ações da rede básica de saúde e da comunidade. Os profissionais que compõem o NASF, entre eles o profissional de EF, devem atuar de maneira integrada e apoiando os profissionais das equipes de Saúde da Família e da Atenção Básica, compartilhando práticas e saberes em saúde com as equipes de referência apoiadas, buscando auxiliá-las no manejo ou resolução de problemas clínicos e sanitários, bem como agregando práticas, na atenção básica, que ampliem o seu escopo de ofertas $^{5,6}$.

Para auxiliar na implementação das DCN com relação às necessidades do SUS, o governo federal publicou os editais do PRÓ-Saúde e PET-Saúde que inclúram diferentes universidades brasileiras em projetos interprofissionais e interdisciplinares ${ }^{7,8}$. O Pró-Saúde tem como objetivo a reorientação da formação profissional, assegurando uma abordagem integral do processo saúde-doença com ênfase na Atenção Básica, promovendo transformações nos processos de geração de conhecimentos, ensino e aprendizagem e de prestação de serviços à população ${ }^{7}$; o PET-Saúde (Programa de Educação pelo Trabalho para a Saúde), considerado uma inovação pedagógica, que agrega os cursos de graduação da área da saúde e fortalece a prática acadêmica que integra a universidade, em atividades de ensino, pesquisa e extensão, com demandas sociais de forma compartilhada ${ }^{8}$.

O PRÓ-Saúde, articulado ao PET-Saúde, é desenvolvido nas redes de Atenção à Saúde, com a participação de docentes, estudantes, equipes de saúde do serviço e usuários do SUS. Os projetos são acompanhados por comissões gestoras locais, constituídas por representantes dos docentes, gestores municipais de saúde, discentes e membros dos conselhos locais. Estes programas têm possibilitado vivências que ampliam a visão de professores, estudantes e profissionais do serviço, sobre o cuidado ao sujeito que adoece no contexto de sua existência, colaborando para a escuta permeada por dúvidas, encantamentos e descobertas do imprevisível, qualificando a atenção à saúde que valoriza a relação entre humanos ${ }^{7,8}$.

Portanto, o perfil dos profissionais da rede de Atenção Básica, entre os quais o da EF, deve contemplar posturas que superem a perspectiva individualizante $\mathrm{e}$ fragmentária que tradicionalmente abordam os modos de viver. Colocam assim, os sujeitos e as comunidades como responsáveis únicos pelas várias mudanças e arranjos ocorridos no processo saúde-doença ao longo da vida ${ }^{9}$.

Uma vez que os profissionais de EF fazem parte da área da saúde, o objetivo do presente estudo foi avaliar se estes profissionais apresentam conhecimento sobre o conceito ampliado de saúde na sua formação acadêmica, pois verifica-se que existem incipientes estudos publicados $^{9-12}$ a respeito deste profissional no ambiente da saúde, do mesmo modo são insuficientes as publicações sobre a atuação de tais profissionais no Sistema Único de Saúde. 


\section{METODOLOGIA}

Trata-se de um estudo transversal de natureza quantitativa. A população do estudo foi composta por profissionais de EF egressos de uma universidade comunitária localizada em Itajaí, no Estado de Santa Catarina, tendo 394 egressos até o ano de 2017/I. Os contatos com os egressos foram realizados por meio de correio eletrônico, sendo os endereços obtidos na Coordenação do Curso após autorização prévia da Vice-reitoria de Graduação da referida universidade.

Para a coleta dos dados, aplicou-se no segundo semestre de 2017 um questionário especialmente construído para a pesquisa com auxílio de uma ferramenta para a criação de formulários "online" do Google Docs. Este instrumento foi fechado, de múltipla escolha, variáveis categorizadas, contendo nove questões referentes a dados sociodemográficos: faixa etária (20 a 25,26 a 30, 31 a 35,36 a 40,41 a 45,46 a 50 e mais de 50 anos), sexo (masculino, feminino), renda (menor que $\mathrm{R} \$ 1.000,00$, de $R \$ 1.000,00$ a menor que $R \$ 2.000,00$, de $R \$ 2.000,00$ a $R \$ 3.000,00$ e maior que $R \$ 3.000,00)$; de formação profissional: carga horária (até 20h, mais de 20h/semanais), anos de formado (até 10 anos, mais de 10 anos), titulação (graduação, especialização, mestrado, doutorado, pós-doutorado), modalidade (licenciatura, bacharelado, licenciatura e bacharelado, licenciatura plena), área de atuação (ensino, condicionamento físico, grupos especiais, performance, saúde e qualidade de vida) e de conhecimento do conceito ampliado de saúde. Esta última questão envolvia três conceitos: o ampliado de saúde ${ }^{1}$, o de promoção da saúde "processo de capacitação da comunidade para atuar na melhoria da sua qualidade de vida e saúde, incluindo maior participação no controle desse processo"13 e o biomédico "A saúde constitui a liberdade de doença, dor, ou defeito, o que torna a condição humana normal saudável" ${ }^{14}$.

A presente pesquisa foi aprovada pelo Comitê de Ética em Pesquisa da Universidade do Vale do Itajaí, sob o parecer 1.713 .302 de 05 de setembro de 2016.

Os dados foram armazenados e tabulados pelo programa Excel e posteriormente as análises realizadas no programa Stata, versão 13.0. As variáveis estudadas foram apresentadas por meio de frequências absolutas e re- lativas. As associações entre o conceito ampliado de saúde e a modalidade de formação foram realizadas utilizando o teste do $\mathrm{x}^{2}$ de Pearson com nível de significância de $5 \%$. Na modalidade formação, separou-se em três grupos: a) licenciatura; b) bacharelado; e; c) cursaram os dois juntos (licenciatura mais bacharelado) e licenciatura plena.

\section{RESULTADOS}

Dos 394 egressos de uma universidade comunitária no Estado de Santa Catarina, 21\% $(n=81)$ retornaram o questionário online preenchido.

A Tabela 1 apresenta o perfil sociodemográfico dos profissionais de EF egressos. Observa-se a predominância de sexo masculino (56\%), faixa etária entre 20 a 25 anos (55\%), carga horária de trabalho semanal de mais de 20 horas (67\%) e renda de até $\mathrm{R} \$ 2.000,00$ (2 mil reais) mensais (78\%).

Tabela 1. Perfil sociodemográfico dos profissionais egressos do curso de educação física, Itajaí, 2017

\begin{tabular}{lcc}
\hline Variáveis sociodemográficas & $\mathbf{N}$ & $\%$ \\
\hline Sexo & & \\
\hline Masculino & 45 & 56 \\
Feminino & 36 & 44 \\
\hline Faixa etária & & \\
\hline $20-25$ anos & 45 & 55 \\
$26-30$ anos & 25 & 31 \\
$31-35$ anos & 11 & 14 \\
Carga horária de trabalho & & \\
Até 20 horas & 14 & 33 \\
Mais de 20 horas & 67 & 67 \\
\hline Renda & & \\
\hline Até $\$ \$ 1.000,00$ & 18 & 22 \\
De $\$ 1.000,00$ a $2.000,00$ & 45 & 56 \\
De $\$ 2.000,00$ a 3.000,00 & 10 & 12 \\
Mais 3.000,00 & 08 & 10 \\
\hline
\end{tabular}

A Tabela 2 mostra a formação dos profissionais de EF egressos. Verifica-se a predominância de até dez anos de formação (86\%), com a modalidade licenciatura (37\%), somente graduação (44\%) e atuando no ensino (43\%). Somente $9 \%$ da amostra atuam na saúde coletiva. 
Tabela 2. Perfil da formação dos profissionais egressos do curso de educação física, Itajaí, 2017

\begin{tabular}{lll}
\hline Formação & N & (\%) \\
\hline Anos de formado & 70 & 86 \\
Até 10 anos & 11 & 14 \\
Mais de 10 anos & & \\
Modalidade & 30 & 37 \\
Licenciatura & 24 & 30 \\
Bacharelado & 16 & 20 \\
Licenciatura + bacharelado & 11 & 13 \\
Licenciatura plena & & \\
Grau de escolaridade & 38 & 47 \\
Graduação & 36 & 44 \\
Especialização & 7 & 09 \\
Mestrado & & \\
Área de atuação & 35 & 43 \\
Ensino & 22 & 27 \\
Condicionamento físico & 09 & 11 \\
Grupos especiais & & 10 \\
Performance & 08 & 09 \\
Saúde e qualidade de vida & 07 & \\
\hline
\end{tabular}

Nota: adiabéticos, hipertensos e idosos

Com relação ao conceito de saúde, verificou-se que $74 \%(n=60)$ dos entrevistados demonstraram conhecimento do conceito ampliado de saúde, $19 \%(\mathrm{n}=15)$ optaram pelo da promoção da saúde, $5 \%(\mathrm{n}=4)$ pelo modelo biomédico e $2 \%(n=2)$ não quiseram responder. Quando se avaliaram os profissionais de EF atuantes na área da saúde coletiva, observou-se que $14 \%$ não apresentavam conhecimento do conceito ampliado de saúde. Com relação aos atuantes em outras áreas, $24 \%$ não possuíam este conhecimento (Tabela 3).

Tabela 3. Conceito ampliado de saúde dos profissionais egressos do curso de educação física, Itajaí, 2017

\begin{tabular}{lcc}
\hline & \multicolumn{2}{c}{ Área de atuação } \\
Conceito ampliado de saúde & $\begin{array}{c}\text { Saúde coletiva } \\
\text { N (\%) }\end{array}$ & Outras N (\%) \\
\hline Correto & $6(86)$ & $54(73)$ \\
Incorreto & $1(14)$ & $18(24)$ \\
Sem informação & $0(0)$ & $02(3)$ \\
Total & $\mathbf{7 ( 1 0 0 )}$ & $\mathbf{7 4}(\mathbf{1 0 0 )}$ \\
\hline
\end{tabular}

Foi realizada a associação entre as variáveis conceito ampliado de saúde e modalidade de formação, nas quais foi observado que os egressos dos cursos de bacha- relado, licenciatura mais bacharelado e plena têm maior conhecimento do conceito ampliado de saúde do que somente os licenciados (TABELA 4).

Tabela 4. Modalidade de formação e o conceito ampliado de saúde dos profissionais egressos do curso de educação física, Itajaí, 2017

\begin{tabular}{lccc}
\hline Conceito & \multicolumn{2}{c}{ Modalidade } \\
\hline & Licenciatura & Bacharelado & $\begin{array}{c}\text { licenciatura/ } \\
\text { Bacharelado + Plena }\end{array}$ \\
\hline Correto & $\mathbf{N}(\%)$ & $\mathbf{N}(\%)$ & $\mathbf{N}(\%)$ \\
Incorreto & $14(47 \%)^{\mathrm{a}}$ & $21(88 \%)^{\mathrm{b}}$ & $23(89 \%)^{\mathrm{b}}$ \\
Total & $\mathbf{3 0 ( 1 0 0 \% )}$ & $\mathbf{2 4}(\mathbf{1 0 0 \% )}$ & $\mathbf{2 7}(\mathbf{1 0 0 \% )}$
\end{tabular}

Teste $\mathrm{X}^{2}$ de Pearson. Letras diferentes diferenças estatísticas com $\mathrm{p}<0,05$.

\section{DISCUSSÃO}

O presente estudo teve como objetivo verificar se o conceito ampliado de saúde é reconhecido pelos profissionais de $\mathrm{EF}$ formados em uma universidade comunitária, bem como descrever o perfil sociodemográfico e de formação destes profissionais.

Com relação ao perfil sociodemográfico dos profissionais egressos de EF da universidade comunitária investigada, observa-se a predominância de sexo masculino, contrário ao encontrado em outro estudo realizado no mesmo Estado com o objetivo de analisar os aspectos de formação continuada de egressos dos cursos de EF da Universidade Federal de Santa Catarina ${ }^{10}$ e em Sobral, no Ceará, que avaliou a inserção de egressos dos Programas Multiprofissionais em Saúde da Família ${ }^{11}$ com predominância do sexo feminino, $58 \%$ e 86\%, respectivamente.

Quanto à faixa etária, os participantes deste estudo apresentaram predominância de 20 a 25 anos, resultado semelhante aos egressos da universidade pública de Santa Catarina ${ }^{10}$. Em Sobral (CE), esta faixa etária foi ampliada, até 30 anos, mostrando ser um grupo de adultos jovens ${ }^{11}$.

Em relação à carga horária de trabalho semanal e renda mensal, os resultados foram semelhantes ao estudo realizado em Santa Catarina ${ }^{10}$ em que os egressos investigados atuavam mais de 20 horas semanais (67\%), com predominância de até três salários mínimos (70\%). 
$\mathrm{Na}$ formação dos profissionais de EF egressos verifica-se a predominância de até dez anos de formação, mostrando um público com pouco tempo de formação, grau de escolaridade predominantemente somente a graduação e apenas 9\% da amostra atuava na saúde coletiva. Em uma revisão da literatura sobre estudos produzidos no Brasil entre 1997 e 2013, investigando características de formação profissional, perfil e competências do profissional de EF para atuação na área da saúde, os autores encontraram importantes avanços na formação em saúde, visando a integralidade e a superação do modelo biomédico $^{12}$. Entretanto, ao abordarem sobre a intervenção do profissional de EF no Sistema Único de Saúde, os mesmos autores apontaram divergências entre o perfil necessário e a formação no ensino superior, demandando o domínio de conteúdos teóricos, técnicas e vivências no campo da saúde coletiva, bem como a integração das instituições formadoras com os serviços de saúde, sobretudo por meio de canais permanentes de retroalimentação e discussão de problemas, avanços e necessidades ${ }^{12}$.

As pesquisas produzidas nos meios científicos, nos últimos anos, a respeito da educação para a saúde, têm mostrado a sua reconhecida importância na formação docente ${ }^{15}$. Considerando a relevância do tema, um estudo identificou e analisou as abordagens de saúde contidas em 55 trabalhos científicos em periódicos, dissertações e teses contidas no Portal da Capes, no período de 2005 a 2015, que discutiram a educação para a saúde nos currículos de formação dos professores ${ }^{15}$. A análise do referido estudo possibilitou identificar que as matrizes curriculares que discutem a temática da saúde apresentam uma visão contraditória ao descrito nos documentos oficiais do ensino e de caráter fortemente biologista, o que leva a formação de profissionais com concepções mais individualistas e sem fazer reflexão sobre a importância de coletivos de modo a estabelecer relações com o social e o cultural ${ }^{15}$.

Em relação ao grau de escolaridade, um trabalho que aborda o perfil de estudantes de pós-graduação na área da $\mathrm{EF}$ apontou que as expectativas destes se centram na atuação docente no ensino superior, o que implica uma não adesão à formação continuada específica para a atuação profissional na área da saúde pública ${ }^{16}$, embora os egressos aqui investigados possuam cursos de pós-gra- duação, sendo 44\% de especialização e $9 \%$ mestrado.

No que se refere à área de atuação do egresso no mercado de trabalho, predominou o ensino, na modalidade licenciatura. Percebe-se que os que concluem o curso nessa modalidade se inserem com mais facilidade nesta área de atuação. Diferentemente do estudo que constatou baixo interesse em atuar no ensino (16\%), em função das dificuldades encontradas, tais como as estruturas precárias das escolas, a baixa renda oferecida ao profissional, bem como a falta de valorização econômica e social ${ }^{10}$.

A ampliação das possibilidades de atuação do profissional de EF para ambientes não tradicionais, como hospitais, equipes multiprofissionais, tais como os Núcleos de Atenção à Saúde da Família, clínicas de reabilitação, grupos de terceira idade, são recentes, mas tem sido muito bem recebida pelos profissionais da área de EF como forma de expansão nas suas atuações. Esta ampliação de possibilidades de atuação profissional pode interferir nas escolhas profissionais, na medida em que muitos profissionais relatam insatisfação com as condições de trabalho, tais como remuneração, integração social nos ambientes de trabalho, entre outros fatores ${ }^{17}$, embora elementos como progressão na carreira, autonomia no trabalho e relevância social das ações impliquem em escolhas e manutenção de atividades laborais.

Em um estudo sobre a importância de equipes multiprofissionais na Estratégia Saúde da Família, com enfoque no papel do profissional de EF, os resultados apontaram que a atuação deste profissional no NASF é reconhecida, entretanto, sua inserção nas equipes multiprofissionais precisa ser consolidada, uma vez constatado seu papel fundamental na prevenção de doenças e promoção da saúde ${ }^{18}$.

Verificou-se o interesse dos egressos dos cursos de bacharelado e licenciatura em EF pela realização de atividades de formação continuada em EF, com destaque comum para cursos de especialização e/ou de mestrado nas áreas de atividade física direcionada à promoção da saúde ${ }^{10}$.

Com relação ao conceito de saúde, objeto principal investigado neste estudo, verificou-se que $24 \%$ dos egressos que não atuavam na área da saúde não têm conhecimento do conceito ampliado de saúde e quando se 
avaliaram os atuantes na área da saúde coletiva foram encontrados $14 \%$. Desse modo, observa-se maior conhecimento sobre o conceito ampliado de saúde por parte dos profissionais atuantes na área da saúde. Tal fato pode ser explicado pela participação profissional ativa no campo da saúde pública, em que as diretrizes e preceitos que regem o SUS se fazem presentes no cotidiano desses profissionais. Outro trabalho aponta que diante das políticas de saúde e da própria inserção da EF, neste campo, houve implicações em mudanças e adequações do perfil deste profissional, incluindo alterações de práticas e, especialmente, de forma de compreensão de saúde condizentes com o que se preconiza no SUS, neste caso, o conceito ampliado de saúde?.

O atual conceito ampliado de saúde diferencia do campo biológico, pois é pensado não apenas do ponto de vista da doença, mas dos aspectos econômicos, políticos e histórico-sociais, da qualidade de vida e das necessidades básicas do ser humano, seus valores, crenças, direitos, deveres e das suas relações dinâmicas e construídas ao longo de todo ciclo da vida e do meio em que convive. É imprescindível, nesse contexto, entender saúde por meio das relações históricas e socioculturais que o indivíduo mantém com o outro e com a comunidade e nas suas formas de convivência com o meio ambiente ${ }^{1}$.

A reformulação do Sistema Único de Saúde está em constante construção, pois este sistema é aberto a novos programas e projetos e, nesse contexto, marcado pela promoção da saúde, abre um campo para atuação do profissional de EF nos serviços de saúde pública, implicando no desenvolvimento de novos conceitos e concepções. Dessa forma, o profissional dessa área, inserido no serviço de Atenção Básica do SUS, deve ter formação em saúde coletiva para que possa agir adequadamente no espaço profissional, levando em consideração as necessidades da população e o contexto na qual está inserido, o que remete à noção ampliada de saúde. Pesquisas realizadas acerca do fazer e pensar do profissional de EF no SUS indicam que a atuação deste profissional nesse campo tem sido, ao longo dos últimos anos, ao menos desde a implantação da Política Nacional de Promoção da Saúde (PNPS) em 2006, cada vez mais alinhada ao que se preconiza na saúde coletiva ${ }^{19}$.

O processo de trabalho do profissional de EF deve ser caracterizado por ações compartilhadas, procurando intervir de forma interdisciplinar, visando à ampliação e ao fortalecimento das intervenções junto das equipes de saúde. Sendo assim, a importância desse profissional na saúde coletiva ainda precisa ser fortalecida, para isso é necessário consolidar sua formação e sua atuação na saúde pública. É nesse contexto que a concepção de saúde ampliada deve ser compreendida por esses profissionais para que sua ação profissional seja voltada para a promoção da saúde.

Os dados aqui apresentados, referentes à compreensão de saúde ampliada dos egressos investigados, sugerem que os mesmos possuem condições conceituais para atuar no campo da saúde pública com conceitos pertinentes da saúde coletiva. No entanto, há que se registrar as diferenças nesta compreensão em relação aos que atuam no campo da saúde pública e coletiva e os que não atuam.

Outro aspecto considerado neste estudo diz respeito ao tipo de formação e o conceito ampliado de saúde. Evidenciou-se que os formados em bacharel tinham compreensão do conceito ampliado de saúde seguido pelos com formação em licenciatura mais bacharelado e licenciatura plena e por fim, os licenciados. Destaca-se que a formação profissional em EF para o campo da saúde pública e coletiva tem sido objeto de estudos e investimentos teóricos ${ }^{19,20}$. Independente da modalidade de formação, tem-se argumentado que, para atuação neste campo profissional, são necessários conhecimentos específicos desta área como, por exemplo, o conceito ampliado de saúde. Embora nas Diretrizes Curriculares Nacionais existam especificidades na formação dos licenciados e bacharéis como, por exemplo, relacionadas à carga horária de determinados conteúdos e estágios curriculares obrigatórios, não é possível sugerir, segundo a literatura consultada, ausência de discussões acerca das concepções de saúde ampliada em qualquer modalidade de formação $0^{20}$.

É pertinente observar, no caso específico dos sujeitos investigados, que a maior parte dos egressos (86\%) tem até dez anos de formação o que coincide com o tempo da publicação da PNPS, documento que insere legalmente o profissional de EF na saúde pública. Além do mais, as últimas DCNs para formação na área foram 
publicadas há 15 anos, fato que implica em reorganização curricular para o campo da saúde pública, por exemplo. Tal fato aliado às ações propostas pelos Ministérios da Saúde e da Educação de reorientação da formação das profissões da saúde, como o Pet GraduaSus, podem ter ocasionado conhecimento maior por parte do bacharelado, na medida em que tal programa faz parte dos processos formativos de todos os cursos do Centro de Ciências da Saúde da universidade onde foram formados. O curso de licenciatura desta universidade, na medida em que está vinculado ao Núcleo das Licenciaturas, não participa desses processos formativos, como o Pet GraduaSus.

O Pet GraduaSus é um programa que promove a inserção de graduandos no cenário das Unidades Básicas de Saúde, incitando saberes e vivências neste campo de atuação. É importante destacar que tais ações são fruto de orientações das Diretrizes Curriculares Nacionais para os cursos da área da saúde, que buscam "efetivar a formação acadêmica e profissional com competências, habilidades e conteúdos contemporâneos, capazes de atuarem, com qualidade e resolutividade no Sistema Único de Saúde"21.

Por fim é possível inferir que a presente pesquisa encontrou associação significativa entre a modalidade de formação e o conceito ampliado de saúde. Os egressos dos cursos de bacharelado, licenciatura mais bacharelado e plena têm maior conhecimento do conceito ampliado de saúde do que somente os licenciados. Estes resultados podem ser explicados e justificados a partir das diferenças entre as DCNs e respectivas organizações curriculares de ambos os cursos.

As principais limitações do presente estudo foram o número reduzido de questionários on line preechidos e a ausência de literatura sobre o tema proposto, visto que se trata de um assunto nacional, não sendo possível comparar com a literatura internacional.

\section{CONCLUSÃO}

O perfil sociodemográfico do profissional de EF egresso do presente estudo mostra um público jovem, predominantemente masculino, empregado e com baixa remuneração, tendo uma formação recente, somente graduação e atuando principalmente no ensino.
Com relação à concepção de saúde apresentada pelos profissionais egressos, $74 \%$ apresentaram adequação ao conceito de saúde ampliado, sendo 86\% atuantes na saúde coletiva. Os dados mostraram ainda que os egressos dos cursos de bacharelado, licenciatura mais bacharelado e plena têm maior conhecimento do conceito ampliado de saúde do que somente os licenciados. Os resultados foram encaminhados ao referido curso para discussão, salientando a importância da atuação do profissional de EF na saúde coletiva e a consolidação da sua formação nesta área para inserção nas equipes multiprofissionais, visto que apesar de ser um campo recente de atuação, a amostra do presente estudo verificou que $14 \%$ dos profissionais que atuam na área da saúde coletiva não possuem o conhecimento do conceito ampliado de saúde.

\section{AGRADECIMENTOS}

Ao ProBIC/Univali.

\section{REFERÊNCIAS}

1. Brasil. Lei $\mathrm{n}^{\mathrm{o}} 8.080$, de 19 de setembro de 1990. Dispõe sobre as condições para a promoção, proteção e recuperação da saúde, a organização e o funcionamento dos serviços correspondentes e dá outras providências. Disponível em http://www.planalto. gov.br/ccivil_03/leis/L8080.htm [2018 set 26].

2. Ceccim RB, feuerwerker L. Mudança na graduação das profissões de saúde sob o eixo da integralidade. Cad Saude Publica, Rio de Janeiro, set/out 2004; $20(5) ; 34$.

3. Brasil. Resolução $n^{0} 7 / 2004$, de 31 de março de 2004. Institui as Diretrizes Curriculares Nacionais para os cursos de graduação em Educação Física em nível superior de graduação plena. Disponível em: http:// portal.mec.gov.br/cnearquivos/pdf/ces0704edfisica. pdf [2018 jun 26].

4. Brasil. Ministério da Saúde. Secretaria de Vigilância à Saúde. Secretaria de Atenção à Saúde. Política Nacional de Promoção da Saúde: PNAPS: revisão da Portaria MS/GM n ${ }^{\circ} 687$, de 30 de março de 2006. Brasília (DF): 2014. 
5. Brasil. Ministério da Saúde. Secretaria de Atenção à Saúde. Departamento de Atenção Básica. Núcleo de Apoio à Saúde da Família. Cadernos de Atenção Básica no 39. Brasília (DF): 2014.

6. Brasil. Ministério da Saúde. Secretaria de Atenção à Saúde. Portaria $\mathrm{n}^{0}$ 256, de 11 de março de 2013. Estabelece novas regras para o cadastramento das equipes que farão parte dos Núcleos de Apoio à Saúde da Família (NASF) Sistema de Cadastro Nacional de Estabelecimentos de Saúde (SCNES). Brasília (DF): 2013.

7. Brasil. Ministério da Saúde. Portaria Interministerial $\mathrm{n}^{0} 3.019$, de 26 de novembro de 2007. Dispõe sobre o Programa Nacional de Reorientação da Formação Profissional em Saúde - Pró-Saúde - para os cursos de graduação da área da saúde. Brasília (DF): 2007.

8. Brasil. Ministério da Saúde. Portaria Interministerial $\mathrm{n}^{\mathrm{o}} 1.802$, de 26 de agosto de 2008. Institui o Programa de Educação pelo Trabalho para a Saúde - PET - Saúde.

9. Scabar TG, Pelicioni AF, Pelicioni MCF. Atuação do profissional de Educação Física no Sistema Único de Saúde: uma análise a partir da Política Nacional de Promoção da Saúde e das Diretrizes do Núcleo de Apoio à Saúde da Família - NASF. Journal Health Sci. Inst. 2012; 30(4): 411-418.

10. Salles WN, Farias GO, Nascimento JV. Inserção profissional e formação continuada de egressos de cursos de graduação em Educação Física. Rev Bras Educ Fis Esporte. 2015; 29(3): 475-486.

11. Melo CNM, Chagas MIO, Feijão JRP, Dias MSA. Programa de residência multiprofissional em saúde da família de Sobral: uma avaliação de egressos a partir da inserção no mercado de trabalho. SANARE. jan/ jun 2012; 11(1): 18-25.

12. Guarda FRB, Silva RN, Correia Junior JLA, Freitas MIF, Santos Neto PM. Intervenção do profissional de educação física: formação, perfil e competências para atuar no Programa Academia da Saúde. Rev Panamazonica Saude. 2014; 5(4): 63-74.

13. Brasil. Ministério da Saúde. Secretaria-Executiva. Secretaria de Vigilância em Saúde. Glossário Temático: promoção da Saúde. Ministério da Saúde. Secretaria-
-Executiva. Secretaria de Vigilância em Saúde. Brasília: Ministério da Saúde, 2012. 48 p. (Séria A. Normas e Manuais Técnicos).

14. Annandale E. The Sociology of Health and Medicine: A Critical Introduction, Polity Press, 1998.

15. Schwingel TCPG, Araújo MCP, Boff ETO. A educação em saúde nos currículos de formação de professores. Transmutare. 2016; 1(1): 126-140.

16. Gentil N. Situação profissional de doutores egressos dos programas de pós-graduação em educação física. 2016. [Dissertação de Mestrado]. Florianópolis: Mestrado em Educação Física da Universidade Federal de Santa Catarina; 2016.

17. Both J, Nascimento JV, Sonoo CN, Lemos CAF, Borgatto AF. Bem-estar do trabalhador docente de educação física da região sul do Brasil de acordo com os ciclos vitais. Rev Bras Educ Fis. Esporte. jan/mar 2014; 28(1): 77-93.

18. Schuh LX, Brand C, Krug SBF, Garcia EL, Gaya AR, Roth MA. Inserção do profissional de educação física nas equipes multiprofissionais da estratégia saúde da família. Saúde. 2015; 41(1): 29-36.

19. Wachs F, Almeida UR, Brandão FFF, organizadores. Educação Física e Saúde Coletiva: cenários, experiências e artefatos culturais. Porto Alegre: Rede UNIDA; 2016. 379 p.

20. Gomes IMG, Fraga AB, Carvalho YM, organizadores. Práticas Corporais no Campo da Saúde: uma política em formação. Porto Alegre: Rede UNIDA; 2015. p. 258.

21. Brasil. Ministério da Saúde. Gabinete do Ministro. Portaria Interministerial $\mathrm{n}^{\circ} 421$, de 3 de março de 2010. Institui o Programa de Educação pelo Trabalho para a Saúde (PET Saúde) e dá outras providências. Brasília (DF): 2010. Disponível em http://bvsms.saude.gov.br/bvs/saudelegis/gm/2010/ pri0421_03_03_2010.html [2018 jun 26].

Recebido em: 21/12/2018 Aceito em: 21/05/2019 


\section{Anexo 1}

\section{QUESTIONÁRIO}

1. Qual a sua faixa etária?

a ( ) 20 a 25 anos

b ( ) 26 a 30 anos

c ( ) 31 a 35 anos

d ( ) 36 a 40 anos

e ( ) 41 a 45 anos

f ( ) 46 a 50 anos

g ( ) mais de 51 anos

2. Qual seu sexo?

a ( ) Masculino

b ( ) Feminino

3. Em qual ano você se formou?

4. Você se formou em:

a ( ) Licenciatura

b ( ) Bacharelado

c ( ) Licenciatura e bacharelado

d ( ) Licenciatura plena

5. Qual a sua formação em educação física?

Assinale com um $\mathrm{X}$ a resposta que corresponder a sua realidade.

a ( ) graduado superior

b ( ) pós-graduado - especializado

c ( ) pós-graduado - mestrado

d ( ) pós-graduado - doutorado

e ( ) pós-graduado - pós-doutorado

6. Qual a sua carga horária semanal de trabalho em atividade de educação física?

a ( ) até 20 horas

b ( ) mais de 20horas

7. Sua renda mensal:

a ( ) menos de $\mathrm{R} \$ 1.000,00$

b ( ) de $\mathrm{R} \$ 1.000,00$ a menos de $\mathrm{R} \$ 2.000,00$

c ( ) de $\mathrm{R} \$ 2.000,00$ a $\mathrm{R} \$ 3.000,00$

$\mathrm{d}(\mathrm{)})>\mathrm{R} \$ 3.000,00$
8. Qual sua área de atuação atual:

a ( ) ensino

b ( ) condicionamento físico

c ( ) grupos especiais

d ( ) performance

e ( ) saúde e qualidade de vida

9. Dentro das alternativas abaixo assinale a que se refere ao conceito ampliado de saúde:

a ( ) A saúde constitui a liberdade de doença, dor, ou defeito, o que torna a condição humana normal "saudável".

b ( ) A saúde é a resultante das condições de alimentação, habitação, educação, renda, meio ambiente, trabalho, transporte, emprego, lazer, liberdade, acesso e posse da terra e acesso aos serviços de saúde. Sendo assim, é principalmente resultado das formas de organização social, de produção, as quais podem gerar grandes desigualdades nos níveis de vida. c ( ) É o "processo de capacitação da comunidade para atuar na melhoria da sua qualidade de vida e saúde, incluindo maior participação no controle desse processo". 